\section{The Redeposition of Copper during Sputtering in a Glow Discharge Lamp}

\author{
F. Blum, P. A. Büger, and N. P. Ferreira \\ National Physical Research Laboratory, C.S.I.R., \\ P.O. Box 395, Pretoria, 0001 South Africa \\ (Z. Naturforsch. 32 a, 785-786 [1977]; \\ received April 25, 1977)
}

Electrically non-conducting materials can be analysed in a glow discharge lamp by mixing them with a conducting powder ${ }^{1,2}$. A certain amount of copper powder, for instance, is added to the ground non-conducting sample and the mixture is pressed into a pellet. The non-conducting material can be regarded as being present in the sample pellet as inclusions in a conducting matrix. It is generally assumed that the whole sample surface is covered by a conducting layer after the pre-burn time, typically 20 seconds. The present work is concerned with scanning electron microscope studies on the redepositioning of sample material during the process of sputtering in a glow discharge.

Small holes, 0,5 $\mathrm{mm}$ in diameter, were drilled into the flat surface of copper dises and were filled up with epoxy resin. Thus the sample prepared represented non-conducting inclusions (epoxy resin) in a conducting matrix. These samples were then

Reprint requests to Dr. P. A. Büger, National Physical Research Laboratory, CSIR, P.O. Box 395, Pretoria 0001/ South Africa.
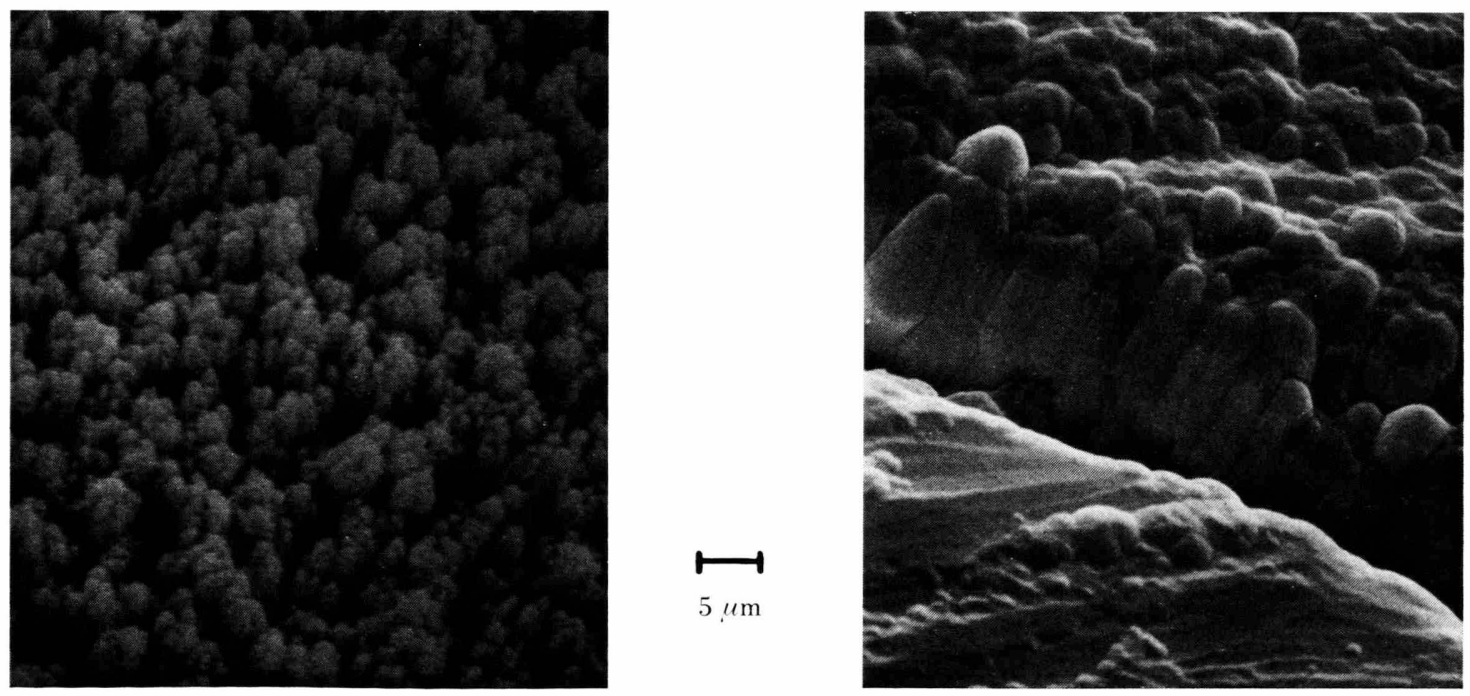

Fig. 1. Secondary electron micrograph of a typical surface area (tilt angle $40^{\circ}$ ) near the centre of non-conducting inclusion. exposed to sputtering in a glow discharge lamp. The electrical discharge parameters were so chosen that excessive heating of the sample was avoided. A discharge current of $20 \mathrm{~mA}$ and a burning voltage of $600 \mathrm{~V}$ were found to be best suited. An increase in the discharge current resulted in the partial melting of the inclusions.

The sputtered surfaces of the samples were studied by means of a scanning electron microscope equipped with an energy-dispersive X-ray spectrometer. Figures 1 and 2 are secondary electron micrographs taken near the centre and near the perimeter of the surface of an "inclusion" (epoxy resin) after the sample had been sputtered for 60 seconds. Both micrographs show that a copper layer had formed over the entire surface of the non-conducting inclusions. A thick porous layer had formed near the centre in contrast with the perimeter where the layer had compacted and was about $15 \mu \mathrm{m}$ thick. The pillar structure of the copper layer is clearly noticeable in Figure 2. Micro-analyses on various areas confirmed that the layer on the inclusions consisted of copper. A shorter sputtering time resulted in thinner layers and it could be concluded that the redepositioning of copper starts at the perimeter of the inclusion. With increased sputtering time the layer develops further towards the centre of the surface of the inclusion.

From the micrographs it is evident that copper is deposited preferentially on the non-conducting inclusions, which is most probably due to the influence of an electric field. The non-conducting areas are negatively charged and will therefore attract the

Fig. 2. Secondary electron micrograph of a typical surface area (tilt angle $40^{\circ}$ ) near the perimeter of non-conducting inclusion. 
positive copper ions present in the discharge plasma. The electric field is stronger at the transition zone between the conducting and non-conducting material giving rise to the gradient in thickness and compactness of the layer on the inclusion.

A rough calculation reveals that about $4 \times 10^{18}$ copper atoms had been sputtered away after $60 \mathrm{sec}$. onds assuming that the energy of the bombarding ions ${ }^{3}$ was about $100 \mathrm{eV}$ and the sputtering yield ${ }^{4}$

1 M. Dogan, Diss., Bochum 1970.

2 S. El Alfy, K. Laqua, and H. Massmann, Z. anal. Chem. 263, 1 [1973].

3 P. W. J. M. Boumans, Anal. Chem. 44, 1219 [1972]. reached 0.5 atoms per incident ion. It is estimated that about $8 \times 10^{17}$ copper atoms, which is $20 \%$ of the copper atoms removed by sputtering, are deposited on the inclusions representing only $1.5 \%$ of the total surface area of the sample disc.

The present contribution provides experimental evidence that an appreciable amount of sample material is redeposited mainly on non-conducting inclusions during sputtering in a glow discharge.

${ }^{4}$ G. K. Wehner, R. V. Stuart, and D. Rosenberg, in Behrisch, Erg. der exakten Naturwissenschaften, Bd. 35, p. 318, Springer-Verlag, Berlin, Göttingen, Heidelberg 1964. 